\title{
Biallelic variants in the ciliary gene TMEM67 cause RHYNS syndrome
}

\author{
Francesco Brancati $\mathbb{1}^{1,2}$ - Letizia Camerota ${ }^{2} \cdot$ Emma Colao $^{3} \cdot$ Virginia Vega-Warner ${ }^{4}$ Xiangzhong Zhao ${ }^{5}$. \\ Ruixiao Zhang ${ }^{6}$. Irene Bottillo $\varpi^{7} \cdot$ Marco Castori $^{8} \cdot$ Alfredo Caglioti $^{9} \cdot$ Federica Sangiuolo $^{10}$ - Giuseppe Novelli ${ }^{10}$. \\ Nicola Perrotti ${ }^{11} \cdot$ Edgar A. Otto $\mathbb{D}^{12}$. Undiagnosed Disease Network Italy
}

Received: 29 October 2017 / Revised: 27 April 2018 / Accepted: 8 May 2018 / Published online: 11 June 2018

(c) European Society of Human Genetics 2018

\begin{abstract}
A rare syndrome was first described in 1997 in a 17-year-old male patient presenting with Retinitis pigmentosa, HYpopituitarism, Nephronophthisis and Skeletal dysplasia (RHYNS). In the single reported familial case, two brothers were affected, arguing for X-linked or recessive mode of inheritance. Up to now, the underlying genetic basis of RHYNS syndrome remains unknown. Here we applied whole-exome sequencing in the originally described family with RHYNS to identify compound heterozygous variants in the ciliary gene TMEM67. Sanger sequencing confirmed a paternally inherited nonsense c.622A > T, p.(Arg208*) and a maternally inherited missense variant c.1289A > G, p.(Asp430Gly), which perturbs the correct splicing of exon 13. Overall, TMEM67 showed one of the widest clinical continuum observed in ciliopathies ranging from early lethality to adults with liver fibrosis. Our findings extend the spectrum of phenotypes/syndromes resulting from biallelic TMEM67 variants to now eight distinguishable clinical conditions including RHYNS syndrome.
\end{abstract}

\section{Introduction}

RHYNS syndrome (OMIM 602152) was defined in 1997 by the acronym of Retinitis pigmentosa, HYpopituitarism,

These authors contributed equally: Letizia Camerota, Emma Colao, Virginia Vega-Warner

Electronic supplementary material The online version of this article (https://doi.org/10.1038/s41431-018-0183-6) contains supplementary material, which is available to authorized users.

Francesco Brancati

francesco.brancati@univaq.it

1 Department of Life, Health and Environmental Sciences, University of L'Aquila, L'Aquila, Italy

2 Laboratory of Molecular and Cell Biology, Istituto Dermopatico dell'Immacolata (IDI) IRCCS, Rome, Italy

3 Medical Genetics Unit, Mater Domini University Hospital, Catanzaro, Italy

4 Division of Nephrology, Department of Pediatrics and Communicable Diseases, University of Michigan School of Medicine, Ann Arbor, Michigan, USA

5 Central Laboratory, The Affiliated Hospital of Qingdao University, Qingdao, China

6 Department of Nephrology, The Affiliated Hospital of Qingdao University, Qingdao, China
Nephronophthisis and mild Skeletal dysplasia in a 17-yearold man [1]. A closely resembling phenotype with liver fibrosis has been previously described in a boy [2], while Hedera and collaborators [3] reported a similar condition in a family with two affected brothers. The observation of affected males only and recurrence in a sibling suggested either an X-linked or autosomal recessive inheritance pattern. Yet, the underlying genetic basis has remained unexplained, although the pattern of associated clinical features was compatible with a hereditary ciliary disorder.

7 Division of Medical Genetics, Department of Molecular Medicine, Sapienza University, San Camillo-Forlanini Hospital, Rome, Italy

8 Division of Medical Genetics, IRCCS Casa Sollievo della Sofferenza, San Giovanni Rotondo, Foggia, Italy

9 Nephrology and Dialysis Unit, Mater Domini University Hospital, Catanzaro, Italy

10 Department of Biomedicine and Prevention, University of Rome Tor Vergata, Rome, Italy

11 Department of Health Sciences, University of Catanzaro Magna Graecia, Catanzaro, Italy

12 Division of Nephrology, Department of Internal Medicine, University of Michigan School of Medicine, Ann Arbor, Michigan, USA 
Fig. 1 Radiological skeletal survey of the proband at age 38 years. a Thickened calvarium. b Short and bowed radius (black open arrow). c Brachydactyly at hands with more severe involvement of middle phalanges, distal phalanx of the thumbs and metacarpals. Generalized reduction of bone density with thinning of the diaphyseal cortex is observed. d Rotoscoliosis. e Short femoral necks (open white arrow)
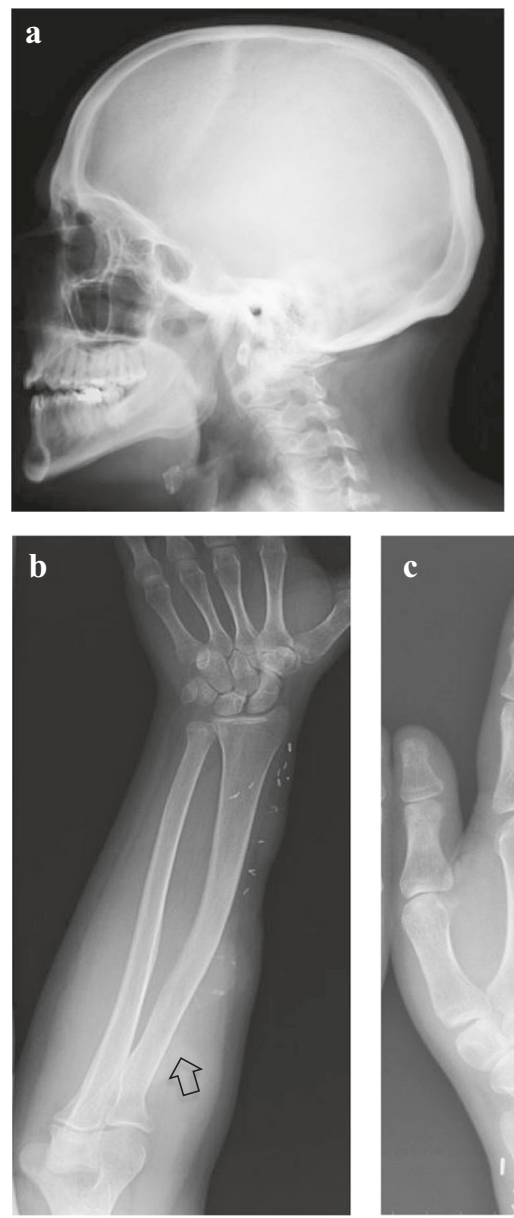
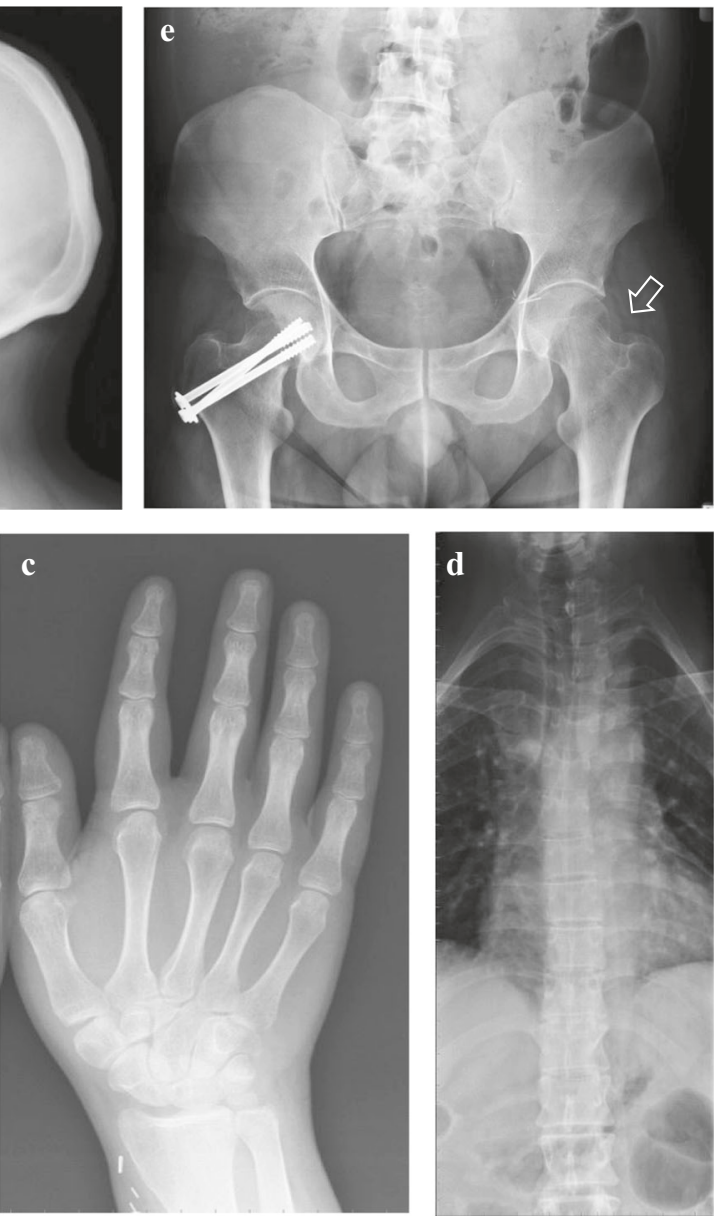

In this study, we reported the 20-year follow-up of the original patient with RHYNS syndrome. Whole-exome sequencing led to the identification of a compound heterozygous variant in the gene TMEM67. The assignment of RYHNS syndrome as part of the wide spectrum of TMEM67-related ciliopathies is discussed.

\section{Subjects and methods}

\section{Patient}

The index male patient was re-evaluated at the age of 38 years, still in search of a molecular diagnosis after receiving a clinical diagnosis of RHYNS syndrome at young age [1]. In brief, he was born with congenital palsy of the III and IV cranial nerves on the right resulting in complete ophthalmoplegia and upper eyelid ptosis and congenital palsy of the VI cranial nerve on the left with exotropia. At the age of 4 years, he measured 90 $\mathrm{cm}(-4.9 \mathrm{SD})$ and showed delayed bone age. Growth hormone and thyreotropin-releasing hormone deficiency were diagnosed and treated with replacement therapy until young adulthood. At this age, radiological examination showed mild signs of skeletal dysplasia consisting of osteopenia, thin tubular bones, epiphyseal hypoplasia and hypoplastic iliac bones with irregular acetabular margins. At age 11, retinitis pigmentosa and left sensorineural hearing loss were first diagnosed. Abnormal renal function was evident by age 12 years, when a renal biopsy demonstrated a histological pattern consistent with nephronophthisis (NPHP). A first kidney transplantation from a deceased donor was performed at age 29 but it was rejected. He was started on hemodialysis and, at age 34, a second renal transplantation was performed. Since then, his clinical condition remained stable.

We have evaluated the patient at age 38 years, when he measured $152 \mathrm{~cm}$ of height with weight $63.5 \mathrm{~kg}$. Generalized and severe osteoporosis was diagnosed by DEXA examination (femoral head: T-Score -3.5 , Z-Score -3.0 , BMD $0.458 \mathrm{~g} / \mathrm{cm}^{2}$; lumbar region: T-Score -3.1 , Z-Score -3.0 , BMD $0.752 \mathrm{~g} / \mathrm{cm}^{2}$ ). A novel skeletal survey detected moderately shortened long bones, bowed radii, short femoral neck, brachydactyly at hands and feet with more severe involvement of middle phalanges, distal phalanx of the thumbs and metacarpals, moderately thickened 
a
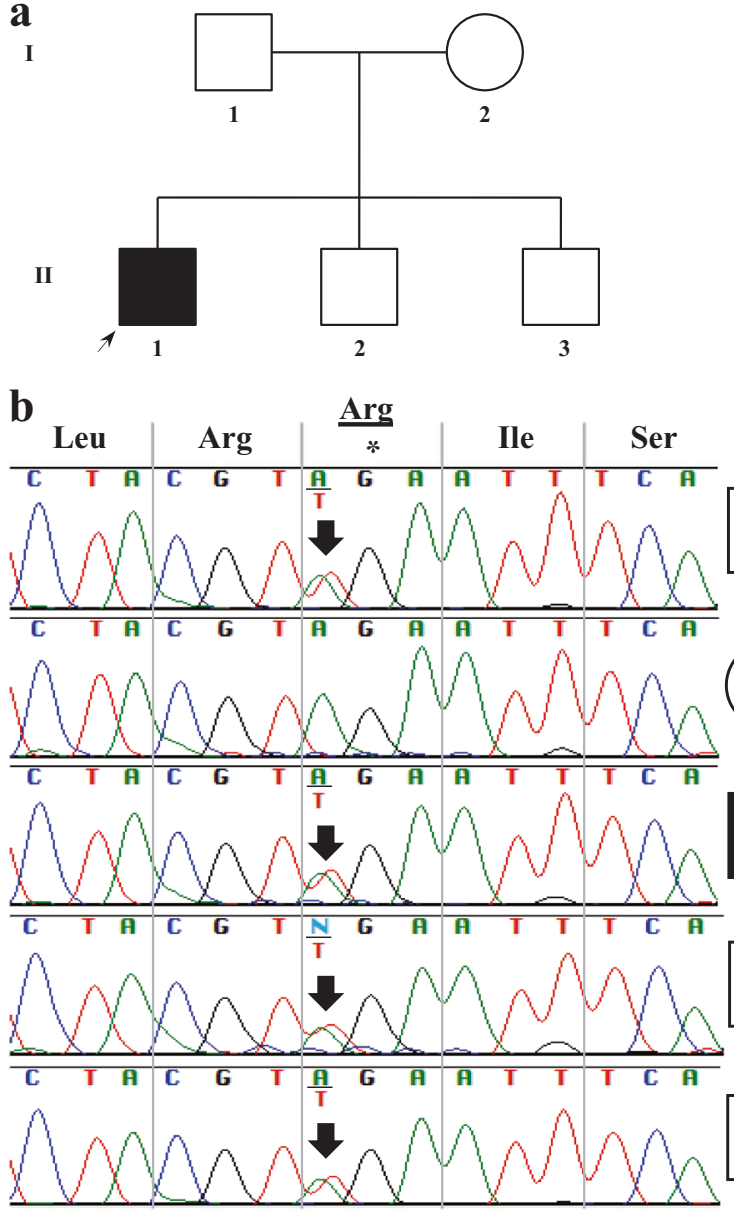

TMEM67 exon 6

c.622A > T, p.Arg208*

Fig. 2 Pedigree of the RHYNS family and TMEM67 electropherograms. a Family tree showing the proband (filled square symbol) and two healthy sibs. Circles and squares indicate females and males, respectively. b Genomic sequence electropherograms demonstrate a nonsense variant (c.622A > T, p.Arg208*) in the father (I-1) and all

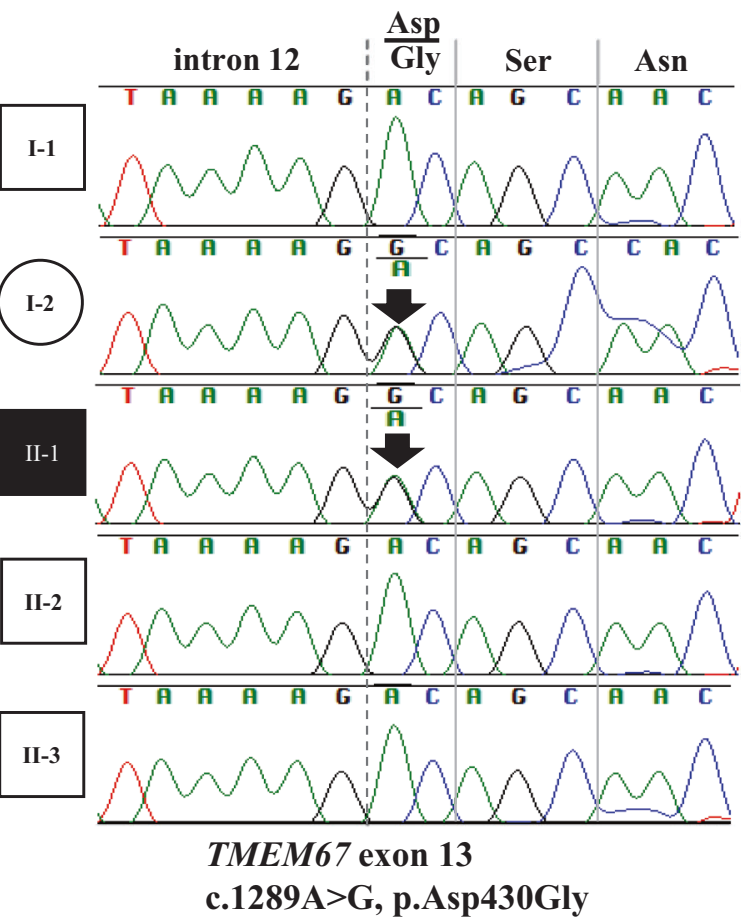

3 sons (II-1, II-2, II-3) and a missense variant (c.1289A > G, p. Asp430Gly) in the affected son (II-1) and his mother (I-2). Arrows indicate a compound heterozygous variant in the affected son and heterozygous changes in all other individuals

calvarium, and rotoscoliosis (Fig. 1d). Diffuse reduction of the bone density with thinning of the diaphyseal cortex, was evident, particularly on hands (Fig. 1b, c).

Hormonal dosage showed increased parathyroid hormone levels in the blood $(164 \mathrm{pg} / \mathrm{ml}$, reference range 14-72 $\mathrm{pg} / \mathrm{ml}$ ), despite having received kidney transplantation, whereas all other hormonal levels were normal, as were liver enzymes and dedicated liver ultrasound.

Audiometry showed pantonal left-sided moderate-tosevere sensorineural hearing loss. Ophthalmologic examination confirmed no residual visual acuity and complete extinguishment of the electroretinogram in both eyes.

Neuropsychological evaluation excluded functional deficits, whereas brain imaging was normal. He obtained the chartered accountant qualification and he was completely

self-sufficient in all daily life activities, although he did not have any current employment.

\section{Whole-exome sequencing}

In order to determine the genetic etiology of the RHYNS syndrome, whole-exome sequencing was performed hypothesizing an-underlying autosomal recessive inheritance pattern. Detailed procedures are available in the Supplementary Methods file.

\section{Mini-gene splicing assay}

Detailed methods are described in the Supplementary Methods file and illustrated in Supplementary Figure 1a. 
Table 1 TMEM67-related phenotypes and distinctive clinical manifestations

\begin{tabular}{|c|c|c|c|}
\hline Syndrome & Major clinical features & $\begin{array}{l}\text { Number of } \\
\text { reported patients }\end{array}$ & $\begin{array}{l}\text { Representative } \\
\text { references }\end{array}$ \\
\hline Meckel syndrome & $\begin{array}{l}\text { - Occipital encephalocele } \\
\text { - Cystic dysplastic kidneys } \\
\text { - Ductal plate malformation } \\
\text { - Hepatic fibrosis } \\
\text {-Postaxial polydactyly }\end{array}$ & 49 & $\begin{array}{l}\text { Smith et al. [5] } \\
\text { Consugar et al. [15] }\end{array}$ \\
\hline Joubert syndrome & $\begin{array}{l}\text { - Molar tooth sign } \\
\text {-Intellectual disability (variable) } \\
\text {-Hypotonia } \\
\text {-Irregular breathing pattern } \\
\text {-Eye movement abnormalities }\end{array}$ & 30 & $\begin{array}{l}\text { Baala et al. [6] } \\
\text { Vilboux et al. [7] }\end{array}$ \\
\hline $\mathrm{COACH}$ & $\begin{array}{l}\text {-Molar tooth sign } \\
\text {-Intellectual disability (variable) } \\
\text {-Ataxia } \\
\text { - Ocular coloboma } \\
\text {-Hepatic fibrosis } \\
\text { - Medullary cystic renal disease } \\
\text {-Nephronophthisis }\end{array}$ & 31 & $\begin{array}{l}\text { Brancati et al. [8] } \\
\text { Doherty et al. [19] }\end{array}$ \\
\hline NPHP11 & $\begin{array}{l}\text { •Nephronophthisis } \\
\text { •Hepatic fibrosis }\end{array}$ & 8 & $\begin{array}{l}\text { Otto et al. [9] } \\
\text { Srivastava et al. [20] }\end{array}$ \\
\hline ARPKD-like & $\begin{array}{l}\text {-Molar tooth sign-like } \\
\text {-Speech apraxia } \\
\text {-Polycystic kidneys } \\
\text {-Nephronophthisis } \\
\text {-Hepatic fibrosis }\end{array}$ & 3 & Gunay-Aygun at al. [10] \\
\hline $\begin{array}{l}\text { Niemann-Pick C } \\
\text { phenocopy }\end{array}$ & $\begin{array}{l}\text {-Molar tooth sign } \\
\text { - Cerebellar atrophy at young age } \\
\text {-Intellectual disability } \\
\text { - Gaze palsy } \\
\text {-Ataxia } \\
\text {-Adolescent-onset dementia } \\
\text {-Hepatic fibrosis }\end{array}$ & 1 & $\begin{array}{l}\text { Tarailo-Graovac et al. } \\
\text { [12] }\end{array}$ \\
\hline $\begin{array}{l}\text { Isolated congenital } \\
\text { liver fibrosis }\end{array}$ & $\begin{array}{l}\text {-Hepatic fibrosis in an otherwise } \\
\text { healthy adult man }\end{array}$ & 1 & Vogel et al. [13] \\
\hline RHYNS & $\begin{array}{l}\text { - Retinitis pigmentosa } \\
\text {-Gaze palsy } \\
\text {-GH- and TSH-deficiency } \\
\text {-Nephronophthisis } \\
\text {-Skeletal dysplasia } \\
\text {-Sensorineural hearing loss }\end{array}$ & 1 & This report \\
\hline
\end{tabular}

ARPDK Autosomal Recessive Polycystic Kidney Disease; $C O A C H$ Cerebellar vermis hypo/aplasia, Oligophrenia, congenital Ataxia, ocular Coloboma, and Hepatic fibrosis; GH growth hormone; NPHP Nephronophthisis; RHYNS Retinitis pigmentosa, HYpopituitarism, Nephronophthisis and Skeletal dysplasia; TSH thyreotropin-releasing hormone

\section{Results}

Two variants in TMEM67 (NM_153704.5; NP_714915.3; MIM\#609884) were identified in the proband, each inherited from a heterozygous parent, consistent with compound heterozygosity and autosomal recessive inheritance. The two identified variants were confirmed in the proband and his parents by Sanger sequencing: the nonsense variant c.622A $>\mathrm{T}$, p. $\left(\operatorname{Arg} 208^{*}\right)$ in exon 6 was inherited form the father, while the missense c.1289A $>\mathrm{G}$ variant, p.(Asp430Gly), near the splice acceptor site of exon 13 was present in the mother (exons are numbered like in NG_009190.1), resulting in a c. [622A $>$ T];[1289A $>\mathrm{G}]$ genotype. The two healthy brothers were carriers of the nonsense variant only (Fig. 2). The missense variant was absent from the 1000 Genomes Project, the Exome Aggregation Consortium (ExAC, http:// exac.broadinstitute.org) and the Genome Aggregation Database (gnomAD, http://gnomad.broadinstitute.org); conversely, the nonsense variant was present at extremely low frequency in the most comprehensive variant database with 49 out of 277,178 alleles reported in gnomAD.

Our hypothesis that the close proximity of the c.1289A $>\mathrm{G}$ variant potentially interferes with splicing was investigated with a mini-gene assay utilizing the pSPL3 vector system. 
Transfection with the wild-type construct resulted in the trapping of exon 13 (387 bp PCR product), whereas the mutant c. $1289 \mathrm{~A}>\mathrm{G}$ construct and the empty vector control lack exon 13 (263 bp PCR product) (Supplementary Figure 1b). Accordingly, one predicted possible outcome of the c.1289A $>\mathrm{G}$ variant is exon 13 skipping, r.(1289_1412)del, and, in this case, the formation of a frameshifted protein, p.(Asp430Serfs*9).

The identified variants affecting TMEM67 were submitted to the LOVD database (URL: https://databases.lovd. nl/shared/genes/TMEM67; patient ID: \# 00155014).

\section{Discussion}

In this study, we report the identification of biallelic variants in the TMEM67 gene as the underlying genetic defect causative of RHYNS syndrome. These findings extend the spectrum of phenotypes resulting from TMEM67 variants to now eight distinguishable ciliopathies (Table 1). Their clinical manifestations display a wide range of presentations ranging from lethal phenotypes to patients with organspecific involvement. The pleiotropic effect of TMEM67 reflects the expression in different tissues during development. In particular, in situ hybridization studies in human embryos have shown that at early gestational age, in addition to discrete brain structures, TMEM67 transcripts were particularly abundant in cartilage primordia, kidney, stomach, and large intrahepatic bile ducts of the liver. At 51-53 days, intense expression was seen in the outer layer of the retina and, later, in cartilage primordia including those of ribs and upper limbs (especially the digits) [4].

Initially, variants in TMEM67 were identified in Meckel syndrome (MKS), a lethal disorder displaying central nervous system malformations, typically occipital encephalocele, multicystic kidneys, ductal plate dysplasia with congenital hepatic fibrosis (CHF) and postaxial polydactyly [5]. Subsequently, Baala and collaborators [6] identified TMEM67 variants in three patients with pure (isolated) Joubert syndrome (JS). Indeed, different subtypes of JS were associated to TMEM67 variants with distinct genotype-phenotype correlations within the spectrum of JS-related disorders (JSRDs), a group of pleiotropic ciliopathies, which share in common the Molar Tooth Sign (MTS) at brain imaging [7]. The strongest correlation was defined with JS and CHF, with $\sim 70 \%$ patients affected by socalled $\mathrm{COACH}$ syndrome (Cerebellar vermis hypo/aplasia, Oligophrenia, congenital Ataxia, ocular Coloboma, and Hepatic fibrosis) carrying biallelic TMEM67 variants [8].

In addition to MKS and JSRDs, 10\% of patients affected by NPHP and CHF without neurological involvement and normal brain imaging (NPHP11; MIM \#613550) had TMEM67 variants [9]. Interestingly, the same gene was also mutated in three children with a unique association of polycystic kidney (mimicking autosomal recessive polycystic kidney disease-ARPKD), NPHP, CHF and midbrainhindbrain abnormalities within the MTS spectrum [10]. Of note, the spectrum of kidney disease in TMEM67 is broad including NPHP, an overlap phenotype of autosomal recessive polycystic kidney disease/NPHP and prenatally enlarged cystic kidneys such as in MKS [11]. More recently, TarailoGraovac et al. [12]. described a young adult patient with two variants in TMEM67, who displayed, in addition to the MTS and cerebellar atrophy at brain imaging, mild intellectual disability, adolescent-onset dementia, vertical gaze palsy, ataxia, and progressive hepatic fibrosis, overlapping Niemann-Pick type C manifestations. At last, TMEM67 was mutated in an otherwise healthy adult patient affected by isolated congenital hepatic fibrosis, which represented so far, the mildest end of the TMEM67-related spectrum [13].

The allelic spectrum of TMEM67 includes missense, truncating and splice site variants, as well as rare multiexon deletions. Two truncating variants (either frameshift, nonsense, or splice site variants) occur with high frequency in MKS and are not reported in non-lethal phenotypes [14]. Conversely, two missense variants or a combination of truncating/splicing and missense variants are prevalent in less-severe phenotypes within the JSRD spectrum, i.e., JS and COACH [8]. Hypomorphic variants in TMEM67 are associated with NPHP and liver fibrosis (NPHP11), whereas more than half of these patients display ocular involvement [9]. Interestingly, our patient carried one truncating and one splicing variant and his phenotype was mainly characterized by retinitis pigmentosa, NPHP without any neurologic involvement or liver fibrosis. The absence of either neurologic or hepatic involvement is surprising since these are major manifestations of TMEM67 variants (Table 1). Among the two variants identified in our patient, only the c.622A > T, p. (Arg208*) was previously reported in three cases of MKS, two in combination with another truncating variant and one with c.755 T > C p.(Met252Thr) missense change [15]. Altogether these observations emphasize the role of, yet unidentified, modifier factors in other genes modulating the penetrance of clinical manifestations. Of note, variants at different loci interacting epistatically under a "multiallelic" inheritance have been proposed as a model for disease penetrance in ciliopathies such as NPHP [16].

In addition, our patient had hypopituitarism without structural abnormalities of the pituitary gland on brain magnetic resonance imaging. Interestingly, growth hormone deficiency was frequently reported in JS caused by variants in two distinct genes (KIAA0753 and CELSR2) in association to absent, small or ectopic pituitary gland, strengthening the importance of ciliary function also in the development of the hypophysis and its function [17, 18].

In conclusion, our data place RHYNS syndrome within the wide spectrum of TMEM67-related ciliopathies, ranging from early lethality to adults with liver fibrosis. More 
studies are encouraged to decipher modifier factors influencing clinical variability.

\section{Availability of data and materials}

All data generated or analyzed during this study are included in this published article.

Acknowledgements We thank the proband and his family for their participation in this study. For technical support, we acknowledge the contribution of Dr. Vincenzo Dattilo and Rodolfo Iuliano from the "Magna Grecia" University of Catanzaro. Funded by grant GR201302356227 to FB.

Author contributions FB and EO had full access to all of the data in the study and take responsibility for the integrity of the data and the accuracy of the data analysis. FB, EO, and NP were responsible for the study supervision. LC drafted the manuscript and interpreted the data. EC collected clinical data. VVW performed sequencing analysis. LC, EC, and VVW equally contributed to the manuscript. XZ, RZ, and IB performed the mini-gene assay and the complementary DNA sequencing. AC is in charge of the patient and contributed relevant clinical data for phenotypic delineation. MC reviewed the skeletal X-Ray images and contributed relevant clinical data for phenotypic delineation. FS, GN, and NP carried out critical revision of the manuscript for important intellectual content. UDNI contributed to the administrative, technical, and material support. All authors contributed to the study concept and design. All authors were responsible for drafting of the manuscript, contributed to the acquisition, analysis, and interpretation of data, read and approved the final manuscript.

Undiagnosed disease network Italy Domenica Taruscio (Rome, Italy); Marco Salvatore (Rome, Italy); Maria Chiara De Stefano (Rome, Italy); Federica Censi (Rome, Italy); Giovanna Floridia (Rome, Italy); Francesco Brancati (L'Aquila, Italy); Giuseppe Novelli (Rome, Italy); Erica Daina (Ranica, Italy); Paraskevas Iatropoulos (Ranica, Italy); Alessandra Ferlini (Ferrara, Italy); Marcella Neri (Ferrara, Italy); Dario Roccatello (Turin, Italy); Simone Baldovino (Turin, Italy); Elisa Menegatti (Turin, Italy); Bruno Bembi (Udine, Italy).

\section{Compliance with ethical standards}

Conflict of interest The authors declare that they have no conflict of interest.

Consent for publication The participants included in the study signed a written informed consent to publish their data.

Ethics approval and consent to partcipate The research protocol, in accordance with the tenets of the Declaration of Helsinki and their reviews, was approved by the Ethics Committee. The patients included in the study signed a written informed consent to participate in the study.

\section{References}

1. Di Rocco M, Picco P, Arslanian A, et al. Retinitis pigmentosa, hypopituitarism, nephronophthisis, and mild skeletal dysplasia (RHYNS): a new syndrome? Am J Med Genet. 1997;73:1-4.
2. Bianchi C, Barera G, Picciotti M, Barbiano di Belgioioso G, Bellini F. Juvenile nephronophthisis associated with new skeletal abnormalities, tapetoretinal degeneration and liver fibrosis. Helv Paediatr Acta. 1988;43:449-55.

3. Hedera P, Gorski JL. Retinitis pigmentosa, growth hormone deficiency, and acromelic skeletal dysplasia in two brothers: possible familial RHYNS syndrome. Am J Med Genet. 2001;101:142-45.

4. Dawe HR, Smith UM, Cullinane AR, et al. The Meckel-Gruber Syndrome proteins MKS1 and meckelin interact and are required for primary cilium formation. Hum Mol Genet. 2007;16:173-86.

5. Smith UM, Consugar M, Tee LJ, et al. The transmembrane protein meckelin (MKS3) is mutated in Meckel-Gruber syndrome and the wpk rat. Nat Genet. 2006;38:191-96.

6. Baala L, Romano S, Khaddour R, et al. The Meckel-Gruber syndrome gene, MKS3, is mutated in Joubert syndrome. Am J Hum Genet. 2007;80:186-94.

7. Vilboux T, Doherty DA, Glass IA, et al. Molecular genetic findings and clinical correlations in 100 patients with Joubert syndrome and related disorders prospectively evaluated at a single center. Genet Med. 2017;19:875-82.

8. Brancati F, Iannicelli M, Travaglini L, et al. MKS3/TMEM67 mutations are a major cause of $\mathrm{COACH}$ syndrome, a Joubert syndrome related disorder with liver involvement. Hum Mutat. 2009;30:E432-42.

9. Otto EA, Tory K, Attanasio M, et al. Hypomorphic mutations in meckelin (MKS3/TMEM67) cause nephronophthisis with liver fibrosis (NPHP11). J Med Genet. 2009;46:663-70.

10. Gunay-Aygun M, Parisi MA, Doherty D, et al. MKS3-related ciliopathy with features of autosomal recessive polycystic kidney disease, nephronophthisis, and Joubert Syndrome. J Pediatr. 2009;155:386-92.

11. Fleming LR, Doherty DA, Parisi MA, et al. Prospective evaluation of kidney disease in Joubert syndrome. Clin J Am Soc Nephrol. 2017;12:1962-73.

12. Tarailo-Graovac M, Shyr C, Ross CJ, et al. Exome sequencing and the management of neurometabolic disorders. N Engl J Med. 2016;374:2246-55.

13. Vogel I, Ott P, Lildballe D, Hamilton-Dutoit S, Vilstrup H, Grønbæk H. Isolated congenital hepatic fibrosis associated with TMEM67 mutations: report of a new genotype-phenotype relationship. Clin Case Rep. 2017;5:1098-102.

14. Iannicelli M, Brancati F, Mougou-Zerelli S, et al. Novel TMEM67 mutations and genotype-phenotype correlates in meckelin-related ciliopathies. Hum Mutat. 2010;31:E1319-331.

15. Consugar MB, Kubly VJ, Lager DJ, et al. Molecular diagnostics of Meckel-Gruber syndrome highlights phenotypic differences between MKS1 and MKS3. Hum Genet. 2007;121:591-9.

16. Hoefele J, Wolf MT, O'Toole JF, et al. Evidence of oligogenic inheritance in nephronophthisis. J Am Soc Nephrol. 2007;18:2789-95.

17. Stephen J, Vilboux T, Mian L, et al. Mutations in KIAA0753 cause Joubert syndrome associated with growth hormone deficiency. Hum Genet. 2017;136:399-08.

18. Vilboux T, Malicdan MC, Roney JC, et al. CELSR2, encoding a planar cell polarity protein, is a putative gene in Joubert syndrome with cortical heterotopia, microophthalmia, and growth hormone deficiency. Am J Med Genet A. 2017;173:661-6.

19. Doherty D, Parisi MA, Finn LS, et al. Mutations in 3 genes (MKS3, CC2D2A and RPGRIP1L) cause COACH syndrome (Joubert syndrome with congenital hepatic fibrosis). J Med Genet. 2010;47:8-21.

20. Srivastava S, Sayer JA. Nephronophthisis. J Pediatr Genet. 2014;3:103-14. 